\title{
مهارة الكلام : تاريخها وكيف نتعلمها ونعلمها
}

\author{
Ahmad Tabrani \\ Universitas Islam Negeri Malik Ibrahim, Malang, Indonesia
}

\section{Email : ahmadtabrani16@gmail.com}

\begin{abstract}
The purpose of this study are to discover the history of speech skills and how to learn and teach them. Students who study Arabic, especially in speech skills, face the problems and difficulties they seek to address. Teachers also do not have a successful teaching ability.

The results of the study are the history of speech skill associated with the emergence of the language, which is divided into three theories: the theory of inspiration and revelation, simulations of natural voices, the theory of agreement and moderation and terminology. And how to learn and teach the skill of speech is to save dialogue and training, and encourage students to speak, and practice Arabic speech, the way of dialogue, and corrected oral errors.
\end{abstract}

Keywords: History of speech skill, method of learning and teaching, practice speech

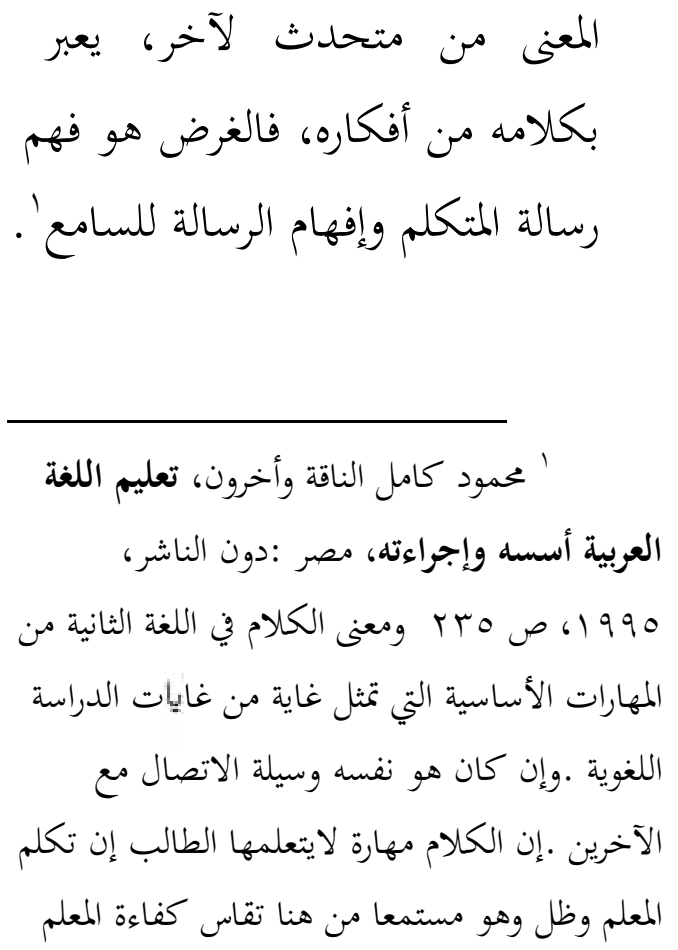


للصغار والكبار. فالناس يستخدمون

الكلام أكثر من الكتابة، أي أهم

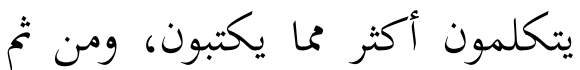
نستطيع أن نعتبر أن الكلام هو اكتران الشكل الرئيسي للاتصال بالنسبة النعان النام للإنسان وأشبع ألوان التعبير وأكثرها قدرة على ترجمة المشاعر بشكل مباشر كما أنه أيضا أكثرها قدرة على ترجمة المشاعر، وأفعلها كوسيلة من وسائل

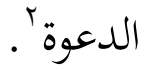

ومن أهمية مهارة الكلام يكتب الباحث حول تاريخ مهارة الكلام وكيف نتعلمها وتعليمها، ولذا يطرح الباحث السؤالان: الأولى كيف تاريخ مهارة الكلام ؟ والثانية كيف تعلم وتعليم مهارة الكلام ؟ وأهداف البحث في هذه الورقة البحثة هي معرفة تاريخ مهارة الكلام ومعرفة كيفية وطريقة تعلم وتعليمها.

$$
\begin{aligned}
& \text { r ' ممود كامل الناقة وآخرون، تعليم } \\
& \text { اللغة العربية أسسه وإجراءثه، ص ب؟T }
\end{aligned}
$$

ومهارة الكلام من مهارات إيجابية

تتطلب من الدارس أن ينتج الأصوات

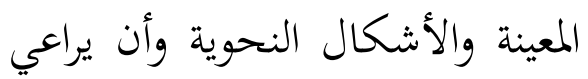

ترتيب الكلمات والجمل حتى تساعده

على التعبير عما يجيش بخلده في

مواقف الحديث المختلفة. وهو الشكل

الرئيسي للاتصال الاجتماعي عند

الإنسان. إذن، يراد بالتعبير الشفوي

أو الكلام هو عملية تبدأ صوتية

وتنتهي باتمام عملية اتصالية تعبر عن

أفكار المتكلم الذاتية. ولهذا يعتبر أن أهم جزء في مهارات اللغة

$$
\text { واستخدامها. }
$$

يقول اللغويون إن الكلام هو

اللغة. فالكالام مهارة لغوية تظهر

مبكرة في حمساة الطفل ولا تسبق إلا

بالاستماع فقط، ذلك الذي من خلاله يعلم الكلام. ولا شك أن الن النان الكلام من أهم ألوان النشاط اللغوي

\footnotetext{
في حصة الكلام بقدار صمته وقدرته على توجيه الحديث وليس بكثرة كلامه.
} 
في العصور القديمة الفيلسوف اليوناني هيراقليط(ت . یی ق م) وفي العصور الحديثة طائفة من العلماء على رأسها لامي والفيلسوف دونالد ومن علماء المسلمين في العصور الوسطى أبو

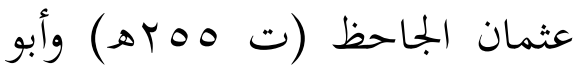

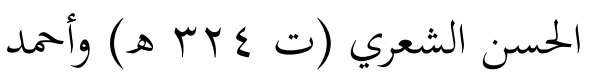
بن فارس (ت 90 هـ هـ) الذي يرى

$$
\text { أن لغة العرب توقيفية". }
$$

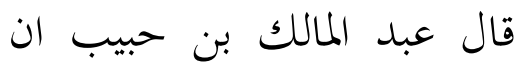

اللسان الأول الذي نزل به أدم من الجنة عربيا، إلى أن بَعُد العهد وطال صار سرانيا، وكان يشاكل اللسان العربي إلا أنه محرف.ويرى علماء

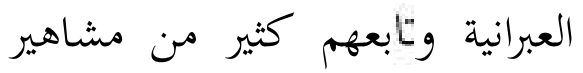
علماء النصرانية وغيرهم أن اللغة العبرانية هي اللغة التي فتق الله بما

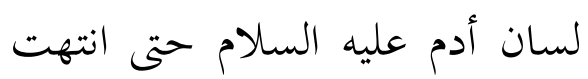
إلى إبراهيم عليه السلام.

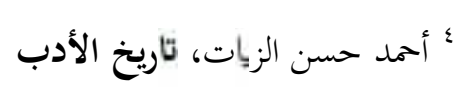

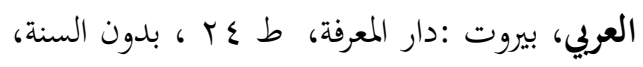
ص

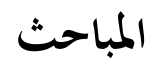
أ. تاريخ مهارة الكلام

اختلف الباحثون قديما وحديثا في موضوع نشأة الكلام الإنساني الأول، ومدى نجاعة دراسة هذا الموضوع بين معارضين للبحث فيه إلى درجة

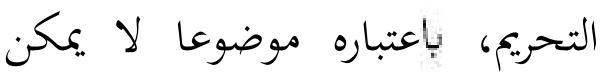
التحقق من صحة وقائعه، وبين مؤيدين بل ومصرين على مثل هذه البحوث اللغوية التي تنبع من التراث المعريّ".

وقد تعددت الأراء

والفرضيات التي تفسر نشأة اللغة الإنسانية الأولى ومن أهها:

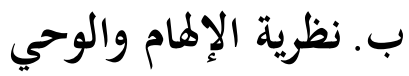
وتذهب هذه النظرية إلى أن الله الخنال أوحى إلى الإنسان الأول وأوقفه على أسماء الأشياء بعد أن الأنسات علمه النطق، وقد ذهب إلى هذا الرأي

$$
\begin{aligned}
& \text { " مصطفى صادق الرافعي، تاريخ أداب }
\end{aligned}
$$

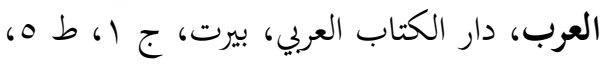

$$
\begin{aligned}
& 07 \text { ص } 1999
\end{aligned}
$$


وتذهب إلى أن أصل اللغة محاكاة

أصوات الطبيعة كأصوات الحيوانات وأصوات مظاهر الطبيعة والتي تحدثها

الأفعال عند وقوعها، ثم تطورت

الألفاظ الدالة على المحاكاة، وارتقت بفعل ارتقاء العقلية الإنسانية وتقدم

الحضارة.

وذهب إلى هذه النظرية ابن جني

قديما وويتني حديثا في القرن 9 احيث إندان

يقول ابن جني وذهب بعضهم إلى أن أصل اللغات كلها، إنما هو أصوات المسموعات كدوي البحر وخرير الماء

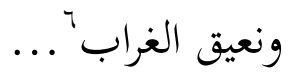

r. نظرية الاتفاق و المواضعة والاصطلاح

تقرر هذه النظرية أن اللغة ابتدعت واستحدثت بالتواضع والاتفاق، وارتجلت ألفاظها ارتجالا،

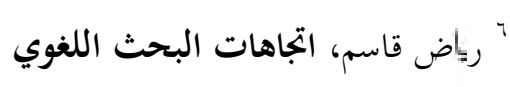

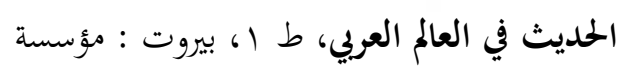

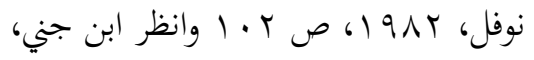

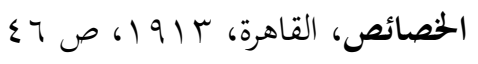

ويعتمد العلماء المؤيدون لهذه

النظرية على ما ورد بهذا الصدد في

العهد القديم من الإنجيل، ويضيفون إلى ذلك ثلاثة وجوه :

أولا، أنه سبحانه وتعالى ذم قوما في إطلاقهم أسماء غير توقيفية في قوله تعالى " إن هي إلا أسماء أنتم وأباوكم ما أنزل الله بها من سلطان....وذلك إلك التهاء يقتضي أن باقي الأسماء توقيفية.

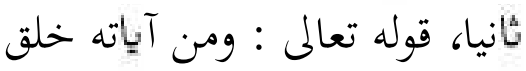
السموات والأرض واختلاف ألسنتكم وألوانكم.... والألسنة غير مرادة لعدم اختلافها، فلمراد هي اللغات. و'الثا هو عقلي. فلو كانت اللغات

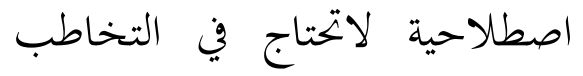

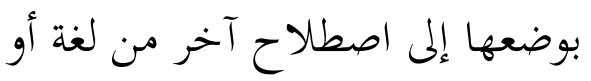
كتابة يعود إليه الكلام، فلابد من إحن الانتهاء إلى التوقيف . 1. محاكات الأصوات الطبيعية

م عبد اللطيف الصوفي، مصادر اللغة في المكتبة العربية، الجزائر : دار الهدى، بدون السنة، or ص 
وتعالى أهلّ الإنسان وأعطاه القدرات الخاصة، فألمهه لكي ينطق وينشئ الاستا اللغة، وبهذه القدرة استطاع الإنسان الأول أن يضع كلماته وبمله الأولى، بالإصغاء والملاحظة والتقليد لما يوجد حوله في الكون، وملا تقدم الإنسان وارتقي في التفكير، بدأ بوضع كلمات جديدة بالتواطؤ والاصطلاح الذي لادي مازال مستمرا إلى يومنا هذا بل إلى الى الى

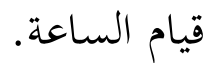

ج. كيفية تعلم و تعليم مهارة الكلام

اللغة في الأساس، هي الككام، أما الكتابة فهي محاولة لتمثيل الكلام،

$$
\text { والدليل على ذلك ما يلي: }
$$

. عرف الإنسان الكلام قبل

أن يعرف الكتابة بزمن الأنسان الكام فيل

طويل، حيث ظهرت الكتابة في فترة متأخرة من تاريخ

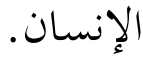

ومال الكثير من العلماء والمفكرين لهذه النظرية منهم الفيلسوف اليوناني ديموقريط وأرسطو والمعتزلة، وقال بهما من المحدين أيضا آدم سميث الانجليزي.

وليس لهذه النظرية أي سند عقلي أو نقلي أو تاريخي بل إن ما تقرره يتعارض مع النواميس العامة التي تسير عليها النظم الاجتماعية، وعهدنا بهذه النظم أهما لا تخلق خلقا بل تتكون بالتدريج من تلقاء نفسها، إضافة إلى ذلك فالتواضع على التسمية يتوقف في كثير من مظاهره على لغة صوتية يتفاهم بهما المتواضعون فبأي لغة لغناهن تواصل هؤلاء”. ومن هنا فإنه لا توجد نظرية واحدة يمكن أن تفسر نشأة اللغة الإنسانية وأن ثلاث نظريات متكاملة يمكن أن تفسر ذلك .فالله سبحانه

" " مصطفى صادق الرافعي، تاريخ أداب العبك، ص ov 
الحاجة إلى هذه المهارة في الفترة

الأخيرة، عندما زادت أهمية الاتصال

الشفهي بين الناس. ومن الضرورة

بمكان عند تعلم وتعليم اللغة العربية،

الاهتمام بالجانب الشفهي، وهذا هو

الابحاه، الذي نرجو أن يسلكه مدرس

اللغة العربية، وأن يجعل همه الأول،

تمكين الطلاب من الحديث بالعربية،

لأن العربية لغة اتصال، يفهمها ملايي

الناس في العالم، ولا حجَّة لمن يهمل

الجانب الشفهي، ويهتم بالجانب

الكتابي، مدعياً أن اللغة العربية

الفصيحة لا وجود لها، ولا أحد

يتكلمهها' .

\section{• تعلم مهارة الكلام بممارسة}

الكلام العربي

إن أفضل طريقة تعلم الطلاب

الكلام، هي أن نعرضهُم لمواقف إنف

تدفعهم لتحدث اللغة. والطالب،

" صالح عبد العزيز وعبد العزيز عبد

الجيديد، التربية وطرق التدريس، مكة :دار المعارف،

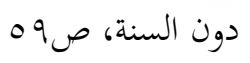

• يتعلم الطفل الكلام قبل أن

يأخذ في تعلم الكتابة، التي

يبدأ في تعلمها عند دخول

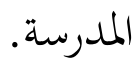

• جميع الناس الأسوياء،

يتحدثون لغاتم الأم بطلاقة،

ويوجد عدد كبير من الناس

لا يعرفون الكتابة في لغاتمم .

• هناك بعض اللغات ما زالت

منطوقة غير مكتوبة.

وبناء على ما تقدم من أسباب،

ينبغي أن نجعل من تعلم وتعليم الكلام

أحد أهم الأهداف في تعلم وتعليم

اللغة العربية.

د. أهمية تعلم وتعليم مهارة

الكام

الككلام من المهارات الأساسية،

التي يسعى الطالب إلى إتقاهَا في

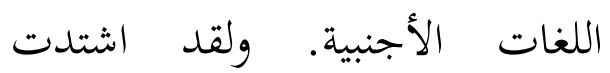

^ حسن شحاتة، تعليم اللغة العربية بين

النظرية والتطبيق،مصر :المكتبة المصرية

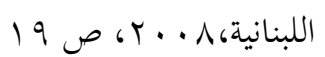


أن يثني على الطالب، كلما كان أداؤه

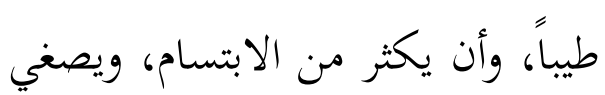
بعناية لما يقوله. إن المطلوب جعل الجو دافئاً في درس الكلام، وتوجيه الطلاب إلى استخدام أسلوب دوبية مهذب، عندما يخاطب بعضهم

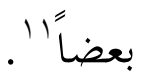

• تعلم مهارة الكلام بحفظ

$$
\text { الحوار وتدريباته }
$$

في المستوى الأول من تعلم اللغة،

تدور تدرييات مهارة الكلام، حول

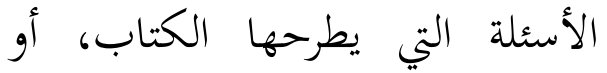

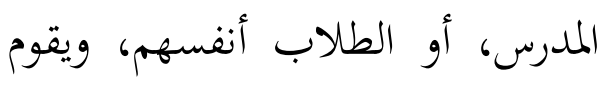

الطلاب بالإجابة عنها. ومن ذلك

أيضاً قيام الطلاب بالثابل بالثدريبات

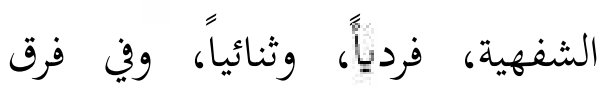

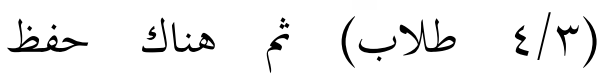

الحوارات وتثيلها. وننصح المدرس بألا

يكلف الطلاب بالكلام عن شيء ونئ المدرس بال

$$
\text { 'ا أحمد فؤاد عليان، المهارت اللغوية }
$$

ماهيتها و طرائق تدريسه ، رياض: دار السلام، ص
ليتعلم الكلام، عليه أن يتكلم. ونود

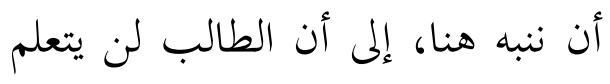

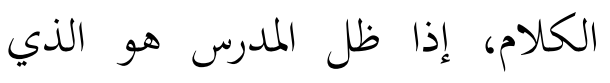
يتكلم طول الوقت، والطالب يستمع. ومن هنا، فإن المدرس الكفء يكون المان قليل الكلام، أقرب إلى الصمت عند

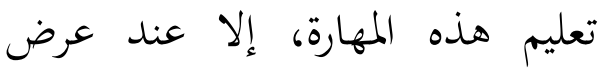

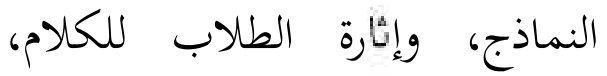

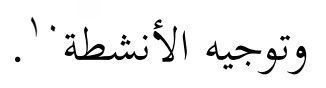

\section{• تعلم مهارة الكلام بتشجيع} الطلاب على الكلام

ينبغي على الطلاب تشجيعا على الى لـابل الكلام، عن طريق منحهم اهتماماً كبيراً عندما يتحدثون، وأن يشعرهم بالاطمئنان، والثقة في أنفسهم، وألا يسخر من الطالب إذا أخطأ، وألا يسمح لزملائه بالسخرية منه. وعليه

$$
\text { " لعبد الرهمن بن ابراهيم الفوزان، }
$$

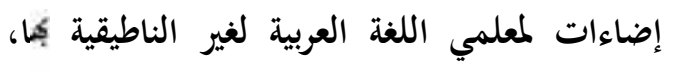
الرياض :فهرسة مكتبة الملك فهد الوطنية أثناء النشر، لكاني Vq ص 6.11 
الطالب لا ينتهي بمجرد استيعاب الحوار وحفظه، وإنما باستخدامه في

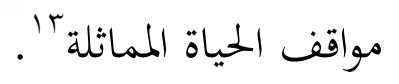

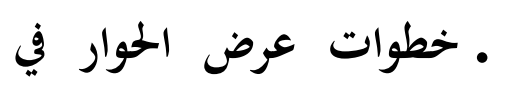

تعليم مهارة الكلام

) التحية: حي الطلاب بتحية

الإسلام, وتلق إجاباهم

عليها.

) إعداد السبورة: اكتب

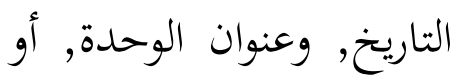

$$
\text { الدرس, ورقم الصفحة. }
$$

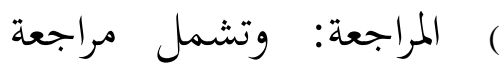

الواجب المنزلي, إن وجد,

ومراجعة الوحدة, أو الدرس إن وجل

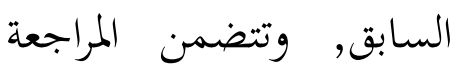

العناصر و المهارات اللغوية,

والمختوى الثقافي.

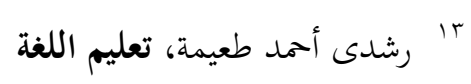

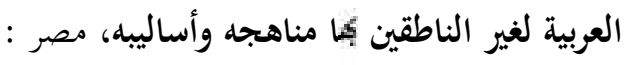

منشورات المنظمة الإسلاميةلتلزبية و العلوم و الثقافة، الثاليه

$$
\text { \&0 ص 61919 }
$$

ليس لديهم علم به، أو ليس لديهم

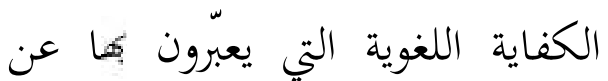

الأفكار التي تطرح عليهم" العائ.

\section{• تعليم مهارة الكلام بالحوار}

للحوار أهمية كبيرة في تعليم اللغة،

فهو غاية ووسيلة في الوقت نفسه:

غاية لأنه الصورة المركزة لمختويات

الدرس، والأساس الذي يمد الطالب الك لأل

بألوان من الجمل والتعبيرات والألفاظ

والأصوات، التي يمتاج إليها الطالب،

وبخاصة عند التدريب على مهارة

الكلام. والحوار وسيلة، لأنه يضم

التراكيب النحوية والمفردات في مواقف

وسياقات ختلفة، تعتمد عليها

التدرييات اللغوية لتأخذ بيد الطالب

نو استعمال اللغة وممارستها في

التعبير والاتصال. وعلى المدرس أن

ينظر إلى الحوار، والثدريبات التي تليه،

باعتبارها كلاً لا يتجزأ، كما أن دور

"' محمود كامل الناقة وآخرون، تعليم اللغة

العربية أسسه وإجراءته، مصر :دون الناشر،

rr人 ص 
) اطلب من الطلاب أداء الحوار

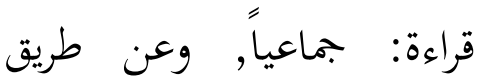

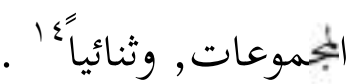

•تعليم مهارة الكلام

\section{بتصحيح الأخطاء الشفهية}

على المدرس ألا يقاطع الطالب

أثناء الكلام، لأن ذلك يعوقه عن

الاسترسال في الحديث، ويشتت

أفكاره، وبخاصة في المستوى الأول.

ومن الأفضل أن نميز بين أمرين:الأول؛

الأخطاء التي تفسد الاتصال، وفي التيز الترن

هذه الحالة، للمدرس أن يتدخل،

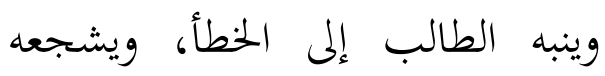

على تصحيحه بنفسه، ما أمكن.

والثاني؛ الأخطاء التي لا تؤثر في فهم

الرسالة، ولكنها تتعلق بشكل الرسالة،

وهذه لا يلح المدرس عليها في المرحلة

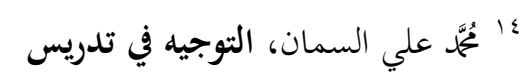

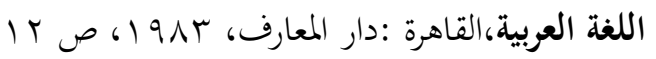

وانظر علي سيد أحم، التعليم والمعلمون،مكة

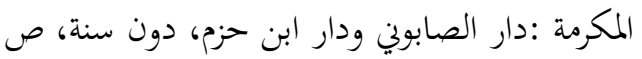

) التمهيد للدرس: ناقش

الطلاب في الصور المصاحبة

للحوار, عن طريق الأسئة.

) المفردات الجديدة: اختر من

المفردات الجلديدة, ما تعتقد

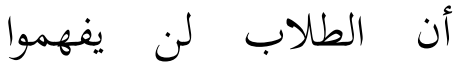

معانيه عن طريق السياق,

وسجلها على السبورة,

وناقش الطلاب في معانيها.

) الاستماع والكتب مفتوحة:

اطلب من الطلاب فتح

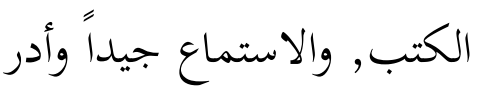

التسجيل, أو أدّ الحوار.

) الاستماع والإعادة: اطلب من الطناون

الطلاب إغلاق الكتب,

وإعادة الحوار بعدك جماعياً,

تم قسم الطلاب إلى

مجموعات, واطلب من كل

مجموعة أن تؤدي جزءاً من واطل

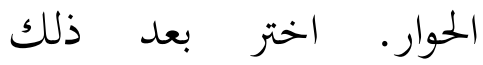

طالبين, لأداء الحوار ثنائياً. 
أن اللغة ابتدعت واستحدثت بالتواضع والاتفاق.

. كيفية تعلم وتعليم مهارة

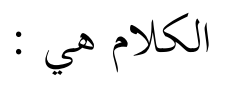

أ.تعلم مهارة الكلام بطريقة

بممارسة الكلام العربي هي

أفضل طريقة تعلم الطلاب

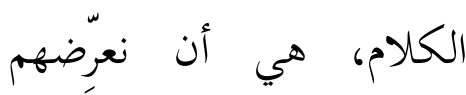

لمواقف تدفعهم لتحدث

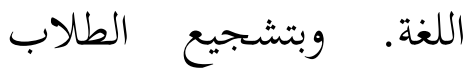

على الكلام ينبغي على

الطلاب تشجيعا على

الكلام، عن طريق منحهم

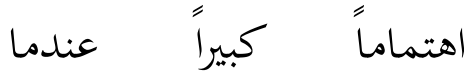

يتحدثون، وبهفظ الحوار

وتدريباته في المستوى الأولى

من تعلم اللغة، تدور تدرييات

مهارة الكلام وحفظ الحوار.

ب. وتعليم مهارة الكلام بطريقة

بالحوار وللحوار أهمية كبيرة

في تعليم اللغة، فهو غاية
الأولى، وإنما يعالجها برفق. إن الطالب

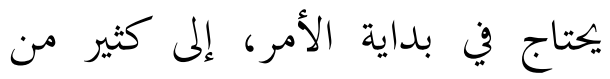

الخاتمة

ـ إن تاريخ مهارة الكلام يرتبط

بنشأة اللغة، وهي تنقسم إلى

ثلاث نظريات : الأولى، نظرية

الإلهام والوحي تذهب هذه

النظرية إلى أن الله الحنال أوحى لإحى

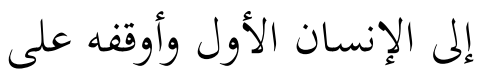

أسماء الأشياء بعد أن علمه

النطق، الثانية،حاكات

الأصوات الطبيعية تذهب إلى

أن أصل اللغة محاكاة أصوات

الطبيعة كأصوات الحيوانات،

والثالثة، نظرية الاتفاق والمواضعة

والاصطلاح تقرر هذه النظرية

10 طه على حسين الداليمي و سعاد عبد

الكريم عباس الوائلى، اللغة العربية مناهجها وطرائق القي

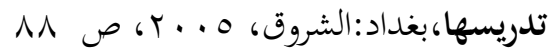


اللبنانية،

$$
\begin{aligned}
& \text { المكتبة المصرية } \\
& r . . \Lambda
\end{aligned}
$$

رشدى أحمد طعيمة، تعليم اللغة العبية لغير الناطقين بها مناهجه

وأساليبه، مصر :منشورات

المنظمة الإسلاميةللتربية و ول

العلوم و الثقافة، 9199

راض قاسم، الجاهات البحث اللغوي

الحلديث في العالم العربي، ط

| 6 بيروت : مؤسسة نوفل6

$$
\text { l. r ص } 6191 r
$$

صالح عبد العزيز وعبد العزيز عبد الميجيدد،

التربية وطرق التدريس، مكة :

$$
\text { دار المعارف، دون السنة }
$$

طه على حسين الداليمي و سعاد عبد الكريم عباس الوائلى، اللغة

العربية مناهجها وطرائق

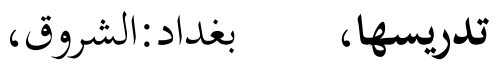

$$
\text { r. o }
$$

عبد الرحمن بن ابراهيم الفوزان، إضاءات لمعلمي اللغة العربية لغير
ووسيلة في الوقت نفسه:

غاية لأنه الصورة المركزة

لمتويات الدرس وتعليم

مهارة الكالام بتصحيح

الأخطاء الشفهية وهي على

المدرس ألا يقاطع الطالب

أثناء الكاءلام، لأن ذلك لك

يعوقه عن الاسترسال في

$$
\text { الحلديث. }
$$

المراجع

ابن جني، الحصائص، القاهرة : دار

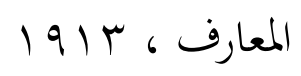

$$
\begin{aligned}
& \text { أحمد حسن الزيات، تاريخ الأدب } \\
& \text { العربي، بيروت :دار المعرفة، } \\
& \text { ط \& ب ، بدون السنة }
\end{aligned}
$$

أحمد فؤاد عليان، المهارت اللغوية ماهيتها

و طرائق تدريسه ، رياض:

$$
\text { دار السلام، بدون السنة }
$$

حسن شحاتة، تعليم اللغة العربية بين

النظرية والتطبيق، مصر 
الناطيقية بجا، الرياض :فهرسة مكتبة الملك فهد الوطنية أثناء

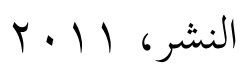

عبد اللطيف الصوفي، مصادر اللغة في المكتبة العربية، الجزائر : دار

$$
\text { الهدى، بدون السنة }
$$

علي سيد أحمد، التعليم والمعلمون، مكة المكرمة :دار الصابوني ودار ابن حزم، دون السنة مُحَّة علي السمان، التوجيه في تدريس اللغة العربية، القاهرة :دار

$$
\text { المعارف، سمبر }
$$

محمود كامل الناقة وآخرون، تعليم اللغة العربية أسسه وإجراءته،

$$
\text { مصر :دون الناشر، } 1990
$$

محمود كامل الناقة وآخرون، تعليم اللغة العربية أسسه وإجراءته،

$$
\begin{aligned}
& \text { مصر :دون الناشر، } 1990 \\
& \text { مصطفى صادق الرافعي، تاريخ أداب } \\
& \text { العرب، دار الكتاب العربي، } \\
& \text { بيرت، ج 1، ط 0، } 1999
\end{aligned}
$$

